\title{
¿TENER MUCHO O DESEAR POCO? DOS APROXIMACIONES AL FENÓMENO ECONÓMICO
}

\author{
ANDREA ETEMADIPOUR ${ }^{\mathrm{a}}$, ANDRÉS NIETO ${ }^{\mathrm{b}}$ \\ ${ }^{\mathrm{a}}$ Universidad Central del Ecuador, ${ }^{\mathrm{b}}$ Universidad París 1 Panthéon-Sorbonne
}

Recepción manuscrito: 1 de julio de 2014

Aceptación versión final: 30 de octubre de 2014

\begin{abstract}
RESUMEN Dentro de la teoría económica neoclásica, existen lagunas con respecto al estudio de la realidad basada en postulados matemáticos y universalizables. Desde la antropología social y más detalladamente desde la antropología económica sustantivista se ha logrado relativizar los postulados económicos, y de manera interesante, el concepto de escasez. Entre los principales autores que han desarrollado esta aproximación se encuentran tres antropólogos: Karl Polanyi, Louis Dumont y Marshall Sahlins. A partir de estos tres autores, indagamos la aproximación antropológica en el estudio de las sociedades tradicionales y de pequeña escala para vislumbrar su interés en la comprensión de la sociedad y del fenómeno económico moderno.
\end{abstract}

PALABRAS CLAVE Economía neoclásica, escasez, antropología económica, ideología, cazador-recolector.

ABSTRACT Inside the neoclassical economic theory, it is possible to find some gaps respect of the study of reality based on mathematical and universal postulates. From social anthropology and, more specifically, from the substantivist economic anthropology it has been possible to achieve a concrete scope of the economic postulates, and, in significant way, of the concept of scarcity. Among the main authors who have developed this approximation we can name three anthropologists: Karl Polanyi, Louis Dumont, and Marshall Sahlins. From them, we look into the anthropological approximation on the study of the traditional and lower scale societies to glimpse its interest on the understanding of society and the modern economic phenomenon.

KEY WORDS Neoclassical economics, scarcity, economic anthropology, ideology, hunter-collector.

JEL CODE B10, B12, Z1

\section{INTRODUCCIÓN}

En el siglo de Copérnico y Galileo, las matemáticas pertenecían al ámbito de lo artificial e impuro, al ser utilizadas por astrólogos, jugadores, arquitectos, pero en ningún caso por científicos. Las disputas teóricas en lo que se refiere al lugar de la Tierra en el universo, culminaron en la exclusión de la divinidad en beneficio del sabio y de las matemáticas para explicar los 
fenómenos observables: desde entonces, el sabio se situará fuera del campo de su objeto, posicionándose como observador neutro. Esta perspectiva neutral y el recurso al lenguaje matemático se reflejarán pocos siglos después en el surgimiento de las ciencias naturales y sociales que al considerar la totalidad de los fenómenos como hechos naturales determinan una realidad objetiva y universal. A modo de ejemplo, en la ciencia económica del siglo diecinueve se teoriza sobre la tendencia reproductiva del ser humano limitada únicamente por los recursos disponibles (Thomas Malthus), la necesidad de subsistir que induce al trabajo (William Townsend) o la teoría ricardiana sobre los rendimientos decrecientes. Uno de los puntos de partida de la cientificidad en Economía, la podemos encontrar en la propuesta teórica de León Walras, que de manera general, pretende convertir la Economía en una ciencia pura con el apoyo de las matemáticas. Sin embargo, la voluntad de universalización de las ciencias sociales y en especial de la ciencia económica, crean problemas de interpretación a la hora de entender sociedades tradicionales o "primitivas» ${ }^{1}$. Estudios realizados desde la historia, la antropología y la sociología han demostrado que la universalidad y objetividad no son características intrínsecas de la ciencia y por lo tanto aplicables a otro tipo de estructuras sociales. A partir de una aproximación comparativa, autores como Polanyi, Bourdieu, Sahlins, Dumont, entre otros, han puesto en duda los axiomas de la economía neoclásica gracias al estudio de los sistemas económicos y el comportamiento de los individuos en las sociedades tradicionales y de pequeña escala ${ }^{2}$. Al comparar el funcionamiento del sistema económico en una sociedad de mercado, con el de estas sociedades, se evidencia la particularidad de ciertas categorías económicas inseparables de una sociedad regida por el mercado, como lo es el concepto de escasez. Erróneamente, como se verá en este artículo, la matematización de la Economía ha otorgado a la teoría económica la autoridad para estudiar todo tipo de sociedad a partir de sus categorías consideradas como hechos naturales.

\section{ECONOMÍA ORTODOXA, NEOCLÁSICOS Y UNIVERSALISMOS}

Desde 1860, Walras intentó convertir a la Economía en una ciencia matemática: su proyecto no era tanto el de describir la realidad como sus predecesores, como el de modelizar la Economía para demostrar sus propios mecanismos de funcionamiento. «Toda esta teoría es una teoría matemática», dirá Walras (1988) en el prefacio de la última edición de su Elementos de economía política pura $^{3}$, lo que le valdrá varios reproches de sus contemporáneos en vista del alejamiento de su modelo de la realidad ${ }^{4}$. Al utilizar el método matemático en economía política, Walras tenía la intención de construir las bases científicas de esta disciplina. Estas pretensiones no pasaron desapercibidas en el mundo de las ciencias sociales, «seguramente no es un accidente el hecho que el ascenso de la economía walrasiana haya coincidido con la virtual matematización de las ciencias sociales» (Rothbard, 1987, p. 97).

Para demostrar las bases de su bloque económico, Walras utiliza el método deductivo con el fin de colocar a la Economía al mismo nivel que las Matemáticas, deduciendo lógicamente sus conclusiones a partir de axiomas previamente expuestos. Al no ser leyes generales inducidas por la experiencia, su teoría no va a tropezar con dificultades de articulación con la realidad, ya que se construyó a partir de tipos ideales que constituyen la esencia de las observaciones. La 
experiencia es considerada únicamente como punto de partida para construir ideales tipo que serán luego la base de un razonamiento lógico alejado de la realidad.Es así como «no se trata de buscar en la experiencia la validación de las conclusiones de la economía pura. Se trata más bien de adoptar la actitud de un ingeniero: frente a un problema concreto, este debe adaptar la teoría pura a una realidad imperfecta» (Lallement, 2000, p. 464). Esta voluntad de erigir primero una teoría para aplicarla en un segundo momento a la realidad es el reflejo de la jerarquización platoniciana de los términos en el pensamiento walrasiano, que concibe a las ideas puras como únicas verdades, al ser éstas superiores e independientes de la realidad que describen. La verdadera ciencia se centrará entonces en el estudio de los hechos ${ }^{5}$, que son permanentes, y no en el de los cuerpos que son efímeros, lo cual ha sido debatido desde la antigüedad con la doctrina filosófica de Heráclito, según la cual lo que se percibe con los sentidos, lo «sensible», es un flujo cambiante e indeterminado. Los cuerpos, cosas sensibles, no conservan eternamente su forma y por esta razón no pueden convertirse en objeto de la ciencia. «Es una verdad desde hace mucho tiempo explicitada por la filosofía platoniciana que la ciencia estudia no los cuerpos, sino los hechos cuyos cuerpos son el teatro. Los cuerpos pasan; los hechos permanecen» (Walras, 1988, p. 39).

La separación de los hechos y su clasificación según si estos dependen de la naturaleza o de la voluntad humana, se refleja en la distinción entre los campos de estudio de la economía política. La economía aplicada se centra en los hechos humanos (relaciones entre humanos y cosas), mientras que la economía pura tendrá como objeto de estudio los hechos naturales (las relaciones objetivas entre las cosas). En este sentido, la economía pura tendrá como principal objeto de estudio el valor de cambio entendido como relación objetiva entre las cosas. Es así como los postulados universales de la economía pura se imponen a la economía aplicada como hechos que no se pueden modificar.

Mientras que el valor de uso sugiere la satisfacción que obtiene un individuo del uso de un bien, lo cual implica la subjetividad de las preferencias del individuo, el valor de cambio es percibido como una propiedad objetiva del bien ya que no depende de los gustos particulares de un individuo, sino que se ve determinado a través del intercambio. Una teoría pura se puede basar únicamente en aquello que es objetivo, de manera que Walras tomará como objeto de estudio de la economía pura el valor de cambio.

Sin embargo, la distinción entre los tipos de valor genera ciertas paradojas: un bien puede tener un importante valor de cambio (un diamante) pero poco valor de uso, o no tener valor de cambio (el aire que se respira) pero un gran valor de uso. Para sobrepasar estas paradojas, los economistas neoclásicos introdujeron la idea de la limitación cuantitativa de un bien: el diamante tienen un valor de cambio elevado a causa de la cantidad limitada de la cual se dispone.

«Digo que las cosas existen a nuestra disposición en cantidad limitada cuando no existen en cantidad suficiente para que cada individuo disponga a discreción para satisfacer enteramente la necesidad que tiene» (Walras, 1988, p. 46). En esta formulación walrasiana de la escasez ${ }^{6}$ (los bienes económicos son útiles y limitados) hay un desplazamiento de la atención hacia la frugalidad de la naturaleza: al basar su definición de la escasez sobre la limitación cuantitativa de un bien, el autor evita plantearse el problema sobre la propensión humana de desear de manera ilimitada ${ }^{7}$. Al plantearse la escasez como relación objetiva entre las cosas, se la considera 
Tabla 1. Diferenciación entre economía pura y economía aplicada

\begin{tabular}{lll}
\hline & ECONOMÍA PURA & ECONOMÍA APLICADA \\
\hline ORIGEN DE LOS HECHOS & Fuerzas de la naturaleza & Voluntad humana \\
OBJETO DE LA CIENCIA & Hechos naturales & Hechos humanos \\
ATRIBUTO & Objetividad & Subjetividad \\
TIPO DE RELACIÓN & Valor de cambio (relación entre cosas) & Valor de uso (relación humanos-cosas) \\
RELACIÓN DETERMINADA POR & Escasez & Preferencias individuales \\
\hline
\end{tabular}

como atributo universalisable de todo bien que se considere útil para el ser humano y que no exista de manera ilimitada. Esta visión es fuertemente criticada desde la antropología sustantivista ya que al considerar la escasez como hecho natural, no pone en duda su existencia ni plantea la pregunta de su surgimiento y uso como categoría.

Esta categoría de escasez explica a su vez la de homo œeconomicus, que constituirá el ideal tipo fundamental de la economía neoclásica, a partir de la introducción de la racionalidad económica. El homo ceconomicus es un ser optimizador a pesar de los límites que se le imponen (como el de la escasez de los recursos naturales) y cada una de sus decisiones implica un cálculo costo-beneficio, necesario para hacer el mejor uso posible de los recursos existentes. Es esta misma limitación de los recursos disponibles que justifica la mera existencia de la Economía como ciencia: la actividad económica se ve justificada por la necesidad de una repartición racional de los recursos. Esto se refleja en la definición contemporánea de Economía, calificada como «ciencia que estudia el comportamiento humano como relación entre unos fines dados y medios escasos que tienen usos alternativos» (Robbins, 1947).

A partir de la conceptualización de la escasez, Walras construye una concepción específica de la naturaleza del ser humano en su dimensión más básica: el hombre está determinado por sus deseos ilimitados, por la avaricia de la naturaleza y su racionalidad aparente. El autor justifica de esta manera el deseo del hombre de mejoramiento constante y propone una solución que a la postre será una de las bases de la economía neoclásica: la producción industrial viene a paliar las escasez «objetiva» de la naturaleza.

Sin embargo, la matematización de la economía y su alejamiento de la realidad trajeron consigo un lastre de conceptos universalizables que tienen la pretensión de ser aplicables para el estudio de todo tipo de sociedad. La economía no toma en cuenta la existencia de otros tipos de sociedades y de sistemas económicos que pueden poner en duda tanto el postulado de la escasez natural de los bienes, como del comportamiento económico racional.

\section{APORTES DESDE LA ANTROPOLOGÍA}

Desde que Adam Smith planteó la «predilección del hombre primitivo por las actividades lucrativas», se generó un desinterés generalizado por el estudio del hombre «primitivo» y la Economía se centró únicamente en el estudio de la sociedad de mercado como un hecho natural y evolutivo, el cual culmina en el pensamiento neoclásico. Este olvido de las sociedades tradicionales y de pequeña escala fue criticado por Max Weber, quién «protestó por el olvido 
de la economía primitiva, realizado con el pretexto de que ésta no tenía relación con la cuestión de los móviles y de los mecanismos de las sociedades civilizadas» (Polanyi, 2007, p. 76).

La Antropología Social probará más tarde la importancia de estudiar estas sociedades para comprender de mejor manera los fenómenos económicos y poner en duda sus categorías, clasificándolas de parciales y postulando la imposibilidad de aplicarlas en el estudio de las sociedades «primitivas» (Dalton, 1961). Karl Polanyi es uno de los autores que introdujo el estudio de sociedades «otras» para comprender así la sociedad moderna y en especial el fenómeno económico en Europa. El estudio de las sociedades primitivas nos permite vislumbrar lo que sería un giro teórico y epistemológico en las ciencias sociales, al postular que la Economía no funciona como esfera autónoma, sino que al contrario, hace parte de las relaciones sociales. Polanyi dirá del sistema económico en general que «es gestionado en función de móviles no económicos» (Polanyi, 2007, p. 77), y comprender así las categorías económicas, ya no como hechos naturales; sino que, por el contrario, nacen en la sociedad en la que se encuentran inmersos.

Solo una forma muy particular de etnocentrismo, disfrazada de universalismo, puede inducir a atribuir universalmente a los agentes la aptitud del comportamiento económico racional y suprimir así la cuestión de las condiciones económicas y culturales del acceso a esa aptitud (erigida de tal modo en norma) y, al mismo tiempo, la de la acción indispensable si se pretende universalizar dichas condiciones. (Bourdieu, 2003, p. 18)

Dentro de la misma Antropología, existen dos tendencias distintas en cuanto a la percepción de los fenómenos económicos: mientras que los antropólogos formalistas ${ }^{8}$ continúan refiriéndose al aspecto económico como registro independiente (atomización de la esfera económica y universalismo de los postulados), los sustantivistas, con Karl Polanyi a la cabeza, han intentado romper con este etnocentrismo. A partir de su definición sustantiva, la economía en una sociedad implica «las formas y las estructuras sociales de la producción, de la repartición y de la circulación de bienes que caracterizan esta sociedad en un momento determinado de su existencia» (Godelier, 1973, p.18), lo cual asume que el tipo de comportamiento y su modelo institucional depende del tipo de sociedad. Así, el comportamiento económico racional es dominante únicamente en una sociedad regulada por el mercado entendido como institución. En las sociedades de cazadores-recolectores por ejemplo, existían otros tipos de estructuras sociales que determinaron (y en algunos casos determinan) la forma de la esfera económica. Estas se rigen conforme instituciones sociales específicas como lo son la reciprocidad y la redistribución, la centralidad y la simetría (Polanyi, 2007).

Es a partir de la caracterización de estos tipos de comportamiento de los sistemas económicos en sociedades nativas, que la antropología económica sustantivista intenta relativizar al mercado como regulador de toda actividad económica, y por añadidura pone en duda el comportamiento económico racional del homo ceconomicus. El valor e interés del análisis reside en la posibilidad de salir de la óptica cerrada de la modernidad, al considerar las condiciones históricas que dieron nacimiento a las categorías económicas, y poner en duda la evidencia y universalidad de las mismas. Además de Polanyi, autores como Louis Dumont y Marshall Sahlins utilizan esta aproximación comparativa para cuestionar los postulados universalistas y etnocéntricos de la economía neoclásica. 


\section{LA IDEOLOGÍA COMO BASE DE LOS SISTEMAS ECONÓMICOS}

Teóricamente, no nos encontramos muy lejos del pensamiento de Bourdieu, para quién «todo lo que la ciencia económica postula como un dato, vale decir, el conjunto de las disposiciones del agente económico que fundan la ilusión de la universalidad ahistórica de las categorías y conceptos utilizados por esta ciencia, es en efecto el producto paradójico de una larga historia colectiva reproducida sin cesar en las historias individuales, de la que sólo puede dar razón el análisis histórico» (Bourdieu, 2003).

Desde este punto de vista, Louis Dumont en Homo cequalis, propone analizar el fenómeno económico y en general la sociedad a partir del concepto de «ideología» entendida como un «conjunto de ideas y valores comunes en una sociedad» y contrastar la «ideología moderna» con lo que denomina «ideología de las sociedades tradicionales» (Dumont, 1999). Mientras que las sociedades tradicionales eran de tipo holista y tenían como valor supremo el orden y la jerarquía (las necesidades individuales se veían subordinadas por las de la sociedad), para la sociedad moderna de tipo individualista, los valores supremos son la igualdad y la libertad (las necesidades individuales subordinan a las de la sociedad).

La visión de la ideología moderna individualista (en el sentido que da un valor supremo al individuo como ente moral) como contexto de aparición de la categoría de «economía» se comprende a partir del tipo de relación existente entre los hombres y las cosas en la sociedad moderna. En las sociedades tradicionales, por ejemplo, se diferencian según Dumont las riquezas mobiliarias de las inmobiliarias. Mientras que las primeras se desprecian por ser simples relaciones entre hombres y cosas, las segundas no se separan del poder político (relación entre hombres). Pero con la ruptura moderna, la riqueza mobiliaria adquiere autonomía y se separa definitivamente la riqueza inmobiliaria del poder político, lo cual da nacimiento a la Economía como campo autónomo, «distinción que las sociedades tradicionales desconocen». De hecho, «[en las] sociedades tradicionales, las relaciones entre hombres son más importantes, más altamente valoradas que las relaciones entre hombres y cosas. Esta primacía se invierte en el tipo moderno de sociedad, en el que, por el contrario, las relaciones entre hombres están subordinadas a las relaciones entre los hombres y las cosas» (Dumont, 1999).

Detrás de la configuración de los distintos sistemas económicos se encuentra claramente una jerarquización de los valores, los cuales determinan las categorías utilizadas para comprender la realidad, así como la comprensión de los fenómenos sociales. Las diferencias entre ideología tradicional e ideología moderna limitan el alcance de las categorías de la economía que surgieron en la sociedad moderna individualista. Según el autor, no se puede ni se debe aplicar estas categorías para comprender los sistemas económicos de las sociedades tradicionales o no modernas. Esto significa que la Economía no es una ciencia "pura», sino que está directamente relacionada con el conjunto de ideas y valores comunes en una sociedad. Al estar hoy en día inmersos en la ideología moderna individualista, es necesario relativizar los valores detrás del individualismo moderno y dirigirse hacia el estudio de las sociedades tradicionales para comprender las categorías económicas, rompiendo con la óptica cerrada de la modernidad. 


\section{LA RELATIVIZACIÓN DE LA ESCASEZ}

Marshall Sahlins (1983), en su muy reconocida obra Economía de la Edad de Piedra, disipa, por primera vez en la literatura antropológica, algunas confusiones sobre las sociedades de cazadores-recolectores, y muestra, al mismo tiempo, el etnocentrismo a través del cual la cultura occidental ha mirado estas sociedades alrededor del mundo. Una de las tesis principales expuestas en este documento, es la relativización de la escasez como forma primigenia de la existencia humana, concepto determinante en la teoría económica neoclásica y la ideología moderna explicitada por Dumont.

Para Sahlins, la concepción del hombre «primitivo» desde la Antropología y las demás ciencias humanas, comparte un mismo error de interpretación. Las ideas y valores occidentales se han impuesto como un recuadro limitado y a la medida de las necesidades teóricas de las ciencias sociales. Los supuestos económicos y antropológicos, nos dan a entender que el hombre primitivo vivía en una situación desesperada, donde su «incompetencia técnica» lo mantenía sumido en el hambre y el trabajo continuo, y donde apenas podía producir «cultura», en otros términos «la llamada "de subsistencia"» (Sahlins, 1983, p. 13). Dentro de la literatura antropológica existe una contrastación constante entre la cultura paleolítica, donde la principal fuente de energía para satisfacer las necesidades fue, desde el punto de vista antropológico, el esfuerzo humano, y el periodo neolítico, donde comenzó el proceso de domesticación de animales, plantas y donde emergió la «cultura».

Si el hombre moderno, con todas sus ventajas tecnológicas, carece todavía de recursos, ¿qué posibilidades tiene entonces este salvaje desnudo con su arco insignificante y sus flechas? Habiéndose atribuido al cazador impulsos burgueses y herramientas paleolíticas juzgamos su situación desesperada por adelantado. (Sahlins, 1983, p. 17)

Sahlins propone superar este «error» de interpretación básico, que se trate del mito del buen salvaje (tan importante para la filosofía política) o de este hombre primitivo miserable obsesionado por la supervivencia, para buscar así una comprensión de las lógicas culturales indígenas. Para lograr este objetivo, se debe superar la confusión entre la pretensión de universalismo de un concepto y su emergencia a partir de una organización social específica: son las instituciones sociales y los valores de una sociedad que los determinan.

En este sentido, la escasez dentro de la teoría económica neoclásica, es una interpretación sesgada sobre la relación entre las necesidades básicas del hombre y los medios utilizados para satisfacerlas. Para el autor, «la escasez no es una propiedad intrínseca de los medios técnicos. Es una relación entre medios y fines» (Sahlins, 1983, p. 17). En la sociedad moderna, entendemos la condición humana como «una tragedia decretada donde el hombre está prisionero de la ardua labor que significa la perpetua disparidad entre sus carencias ilimitadas y la insuficiencia de sus medios» (Sahlins, 1983, p. 13). Los antropólogos sustantivistas conciben la escasez no como un dato incuestionable de la realidad humana, sino como una construcción dependiente de los sistemas culturales, de las organizaciones simbólicas y sociales. Retomando la reflexión de Alain Caillé, que sigue en este punto a Baudrillard, «la escasez es un efecto y al mismo tiempo una causa, y en este sentido no antecede los sistemas sociales como una matriz primaria» (Caillé, 2001, p. 120; traducción de los autores). La escasez como concepto es una 
construcción cultural propia a occidente. El autor desarrolla una aproximación diferente al estudio de las prácticas de los cazadores-recolectores para así superar las prenociones forjadas alrededor de estas culturas. A diferencia de lo que dicta el «sentido común», el autor demuestra que estas sociedades son las primeras sociedades de opulencia. Para esto, es importante comprender los dos caminos existentes para llegar a la satisfacción de las necesidades:

- Produciendo mucho: La economía de mercado se basa en el supuesto que las necesidades son infinitas, mientras que los medios son limitados, esto genera una brecha entre medios y fines que puede reducirse mediante la productividad industrial.

- Deseando poco: Es lo que el autor llama el «camino zen». «Las necesidades humanas son finitas y escasas y los medios técnicos inalterables pero por regla general, adecuados».

La vía del cazador-recolector es en este punto, diametralmente opuesta a las «exigencias» materiales de los individuos modernos. Este modo de vida se sustenta sobre dos pilares esenciales de opulencia. En primer lugar, existe en las culturas nómadas «una especie de abundancia material» (Sahlins, 1983, p. 22). Los cazadores-recolectores producen utensilios, herramientas y objetos de uso cotidiano con mucha facilidad y que no implican una tecnología avanzada. Esta tecnología es compartida por todos sus integrantes en una especie de «sociedad del conocimiento libre». Los materiales utilizados en su producción se los encuentra en las inmediaciones del hogar y están a disposición de quien desee utilizarlos: piedras, huesos, madera, pieles, etc. «Agregad a esto las costumbres liberales de compartirlo todo, por las cuales los cazadores tienen una merecida fama, y tendréis que toda la gente puede participar en general de la prosperidad existente, tal como sucede en realidad» (Sahlins, 1983, p. 23). Esta abundancia «objetiva» (en cuanto a los medios de producción) es acompañada por una abundancia «subjetiva». En las sociedades primitivas hay una elección social en cuanto a los bienes que desean acumular (Caillé, 2001, 2002).

En efecto, el modo de vida generalizado en las sociedades de cazadores-recolectores es el nomadismo: son pueblos que viajan dependiendo de las estaciones climáticas y la disponibilidad de recursos para su supervivencia. Bajo este contexto, la posesión excesiva de bienes materiales dificulta el traslado y las exigencias de la vida nómada. «Del cazador se suele decir con propiedad que su fortuna es una carga» (Sahlins, 1983, p. 24). Y a diferencia de la conducta del hombre moderno y su propensión al ahorro, la principal característica de la conducta económica de los cazadores es la prodigalidad (inclinación a consumir rápidamente todas las reservas, libres de las obsesiones de la escasez). El sentido de escasez y abundancia toman un giro inesperado: no es la inadecuación del deseo ilimitado y los recursos limitados que crean escasez, sino un sentido práctico y autorregulado que produce abundancia en desmedro de la riqueza y la acumulación. Son muchos los ejemplos expuestos por Sahlins y otros autores (Clastre, 1978; Lizot, 1977; entre otros) que muestran este fenómeno: los sianes de Nueva Guinea, una vez introducida el hacha de metal por los occidentales, aumentan la productividad general de sus medios de subsistencia, sin embargo no utilizan el excedente para incrementar la producción sino que la utilizan para aumentar el tiempo de fiesta y ocio. (Godelier, 1964, p. 122).

Como lo señala Caillé, son varios años de investigaciones antropológicas que sacan a la luz otro aspecto fundamental de las sociedades primitivas: Para atender sus necesidades básicas, 
estas culturas dedican un tiempo mínimo a las tareas de subsistencia (alimentación y preparación de alimentos), dejando, como hemos señalado anteriormente, una importante parte de su tiempo al ocio y al sueño. Las culturas descritas por Sahlins nos dan una clara muestra del poco esfuerzo que necesitan para alimentarse. Los estudios realizados por McCarthy y McArthur (1960) en Arnhem Land, noroeste australiano, muestran que en promedio los individuos de la zona dedican entre cuatro y cinco horas por día a obtener y a preparar alimentos. Sin embargo esta actividad se realiza en intervalos espaciados y los individuos no lo consideran como una actividad penosa. El resto del tiempo lo dedican al reposo. En otro caso de estudio, Richard Lee (1979), muestra el modo de vida de los bosquimanos con respecto al tiempo dedicado al trabajo: en promedio dedican dos horas y nueve minutos por día a tareas de caza o recolección. Su alimentación es variada y rica en nutrientes, a diferencia de las crónicas de misioneros y conquistadores, que tuvieron la tendencia de ver en estos pueblos hambre y miseria: la ración de alimentos diaria supera las 2100 calorías por individuo.

En contra de las nociones divulgadas por la economía neoclásica, la escasez no es una relación objetiva entre medios y fines, sino una construcción socialmente determinada que en algún punto de la historia de la humanidad, se regía por ideas, valores y prácticas que creaban abundancia entre sus miembros. En el mundo moderno, la extrema pobreza y la extrema riqueza pueden ser vistas como resultado de un sistema social, económico y sobre todo ideológico, que determina que la disparidad entre medios y fines puede ser resuelta gracias a la producción y al consumo, donde «todas las cosas deseables están al alcance del hombre, pero nunca enteramente al alcance de su mano» (Sahlins, 1983, p. 16).

\section{CONCLUSIÓN}

La pretendida universalidad y objetividad de la economía neoclásica determina en gran medida la posibilidad a través de la cual nuestra sociedad puede pensarse a sí misma. Desde la concepción etnocéntrica del pensamiento Occidental, la sociedad contemporánea es la culminación de un proceso deseable y casi escatológico, si pensamos en la «miseria» intrínseca de las sociedades nativas. El estudio de sociedades tradicionales y de pequeña escala, desde la Antropología, la Historia y la Sociología, nos permite entender los valores e ideas que como ciencia, la teoría económica dominante, ha proyecto sobre el estudio de poblaciones locales alrededor del mundo. Tanto Dumont y su estudio sobre la sociedad tradicional (la sociedad de castas hindú), como Sahlins y el estudio de sociedades aún más lejanas a las nuestras como son las sociedades de cazadores-recolectores, nos permiten poner en evidencia la ideología que permea nuestra manera de analizar la sociedad y sus fenómenos económicos. Teóricamente, la economía neoclásica limita nuestra comprensión de la situación económica, social y ecológica actual y nuestras posibilidades futuras como sociedad globalizada. Retomando la relativización de la escasez desde la antropología sustantivista, encontramos que en algunas sociedades indígenas el sentido de abundancia se ve completamente trastocado por la forma de vida de sus miembros. La regla oculta de estas sociedades parece ser el de «No desear es no carecer». Estas mal llamadas sociedades «primitivas» pueden, si dejamos de lado los prejuicios del etnocentrismo, marcar el paso hacia un modelo alternativo 
de relacionamiento entre Hombre y medios de subsistencia. Las reflexiones de André Gorz (1994) son esclarecedoras en este punto: la autolimitación como proyecto social y político pueden abrirnos un campo de acción individual y social. Para este autor es determinante que el ser humano contemporáneo regule su esfuerzo para obtener, de manera simple, sus medios de reproducción y subsistencia básicos y limitar así su impacto en un mundo cada vez más complejo e imprevisible.

Desde un punto de vista metodológico, la antropología económica nos deja también sus enseñanzas. El estudio económico ganaría en profundidad y en precisión al considerar otros determinantes de la actividad económica. Los valores, ideas e instituciones de una sociedad podrían saltar a la luz si consideramos la diversidad de perspectivas disciplinarias. La comparación y la profundidad del análisis histórico y comparativo nos pueden dar la medida de las limitaciones de las categorías y conceptos comúnmente utilizados por la ciencia económica. En este sentido, la importancia de una perspectiva interdisciplinaria para estudiar los fenómenos económicos como parte de realidad compleja y no como parte de una esfera artificialmente separada, se refleja en la diversidad de críticas a la economía neoclásica.

\section{NOTAS}

1 El término «primitivo» es usado, de manera general, en la literatura de la antropología económica de las décadas de los años 50 y 60 . Entendemos que este término puede estar desactualizado dentro del campo de la antropología, sin embargo lo utilizamos en ciertos momentos de la argumentación ya que explicita una parte de los debates teóricos que surgieron en esta disciplina.

2 Es decir, en aquellas sin un órgano de Estado (Sahlins, 1976, p. 240).

3 Walras fue reclutado por la Universidad de Lausanne en 1870, donde dispuso de cierto tiempo para sus investigaciones y para realizar lo que sería su Elementos de economía política pura. Esta obra se publicó por primera vez en 1874 .

4 Los Elementos de economía política pura se dividen en 48 lecciones: los primeros se centran en las definiciones utilizadas para la construcción de su teoría del equilibrio general. El autor se inspira del modelo de la mecánica, ya que como la gravedad universal, el equilibrio de los mercados es estable a pesar de las perturbaciones que puedan afectarlo. Si su teoría tuvo una gran influencia, especialmente a inicios del siglo XX y produjo una vasta literatura crítica y largos comentarios, el origen de las definiciones sobre las cuales se construyó su modelo han sido generalmente aceptadas.

5 Estos hechos, objeto de la ciencia, son de dos tipos: 1) Los hechos naturales, que serán el objeto de la «ciencia pura natural» y que provienen de las fuerzas de la naturaleza, que son para Walras «ciegas y fatales». Estos hechos existen por necesidad y se encuentran fuera de alcance de la voluntad humana; y 2) La segunda categoría concierne los hechos que provienen de la voluntad humana, «fuerza clarividente y libre»: se trata de los hechos humanos. Estos fenómenos humanos serán el objeto de la «ciencia pura moral o historia» (Walras, 1988, p. 40).

6 Otra consecuencia de la escasez de un bien es el deseo que tiene el hombre de acrecentar su disponibilidad efectiva y por lo tanto multiplicar su número gracias a la industria. Para Walras, únicamente las cosas raras son industrialmente producibles y multiplicables. La noción de escasez tiene un sentido científico para Walras, como la velocidad en mecánica: no se opone a la abundancia ya que el término se apega a todo bien si éste es útil y existe en cantidad limitada. «[...] A pesar de su abundancia, un bien es raro en política, si es útil y limitado en cantidad» (Walras, 1988, p. 46). Pasamos entonces de la escasez, que proviene de una relación entre necesidad y cantidad, al valor de cambio que es una relación entre cosas, y la industria que es una relación entre hombre y cosas. 
7 Uno de los principales admiradores de Walras, Schumpeter, concebía metodológicamente errado que la Economía se interese en la «conducta económica» y en «las motivaciones de la conducta humana» (Machlup, 1951).

8 Los partidarios del formalismo van a utilizar una definición clásica de la Economía, aquella propuesta por Lionel Robbins. Para este autor, la Economía es considerada como la «ciencia que estudia el comportamiento humano como una relación entre fines y medios raros que tienen usos alternativos» (Robbins, 1947). El término economía es tomado de esta manera en el sentido de economía de medios, es decir de medios limitados para fines ilimitados, lo cual hace referencia a la ley de maximización y a la noción de escasez utilizadas en Economía. Según esta definición, los medios que dispone todo grupo social son postulados como raros o escasos y es a partir de ciertas estrategias utilizadas (homo oeconomicus), que el grupo logra maximizar sus ganancias. Esta concepción, que erige al grado de universal al homo œeconomicus, va a ser fuertemente criticada por los sustantivistas, en vista de que no toma en cuenta el hecho de que tales comportamientos podrían derivarse de la organización social y no ser inherentes a la naturaleza humana.

\section{BIBLIOGRAFÍA}

Abraham, Y. (2005). La conception naturaliste du marché dans la pensée économique, d’Adam Smith aux néoclassiques. En HEC Montréal, Cahier de recherche, (05-04).

Bourdieu, P. (2003). Las estructuras sociales de la Economía. Barcelona, España: Editorial Anagrama.

Caillé, A. (2001). La rareté reconsidérée. Revue du MAUSS, 18(2), 119.

Clastres, P. (1978). La sociedad contra el Estado. Barcelona, España: Monte Avila.

Crosby, A. (1997). The Measure of Reality: Quantification and Western Society, 1250-1600. Cambridge, England: Cambridge University Press.

Dalton, G. (1961). Economic theory and primitive society. American Anthropologist, 63, 1-25.

Drouin, J. (2006). Les grands économistes. Paris, France: PUF.

Dumont, L. (1999). Homo aequalis. Génesis y apogeo de la ideología económica. Madrid, España: Taurus.

Godelier, M. (1964). Economie politique et anthropologie économique (à propos des Siane de NouvelleGuinée). L'Homme, 4(3), 118-132.

Godelier, M. (1973). Sur les sociétés précapitalistes. Paris, France: Ed. Sociales.

Gorz, A. (1994). Ecología política. Expertocracia y autolimitación. Nueva Sociedad, 134, 32-41.

Lallement, J. (2000). Prix et équilibre selon Léon Walras. En Béraud y Faccarelo (Eds.) (2000). Nouvelle Histoire de la pensée économique 2. (p. 449-497). Paris, France: La découverte.

Lee, R. B. (1979). The !Kung San: Men, women, and work in a foraging society. Cambridge, England: Cambridge University Press.

Lizzot, J. (1977). Population, ressources et guerre chez les Yanômami. Libre, 77, 2.

McCarthy, F. y McArthur M. (1960). The Food Quest and the Time Factor in Aboriginal Economic Life. En Mountford C. P. (Ed.). Records of the AustralianAmerican Scientific Expedition to Arnhem Land, vol. 2: Anthropology and Nutrition. Melbourne, Australia: Melbourne University Press.

Malthus, T. (1846). Ensayo sobre el Principio de la Población. Madrid, España: Lucas González.

Polanyi, K. (2007). La gran transformación: Los orígenes políticos y económicos de nuestro tiempo. México, Buenos Aires: Fondo de Cultura Económica.

Robbins, L. (1947). Essai sur la nature et la signification de la science économique. Paris, Francia: Médicis. Rouge-Pullon, C. (1996). Introduction à l'oeuvre de Walras. Paris: Ellipses.

Rothbard, M. (1987). Breaking Out of the Walrasian Box: The Cases of Schumpeter and Hansen. Review of Austrian Economics, 1, 97-108. 
Sahlins, M. D. (1983). Economía de la edad de piedra. Madrid, España: Akal.

Sahlins, M. (2008). La nature humaine, une illusion occidentale. France: Editions de l'éclat.

Walket, D. (2000). Les Idées de Léon Walras sur la Nature Humaine. En Béraud et Faccarelo (Eds.), Nouvelle Histoire de la pensée économique 2 (p. 104-133). Paris, France: La découverte.

Walras, L. (1988). Eléments déconomie politique pure. Paris, France: Economica. 